Journal of Engineering and Applied Sciences 14 (13): 4448-4452, 2019

ISSN: 1816-949X

(C) Medwell Journals, 2019

\title{
Detection of $\beta$-Lactam Resistance PER-1 Gene in Multidrug Resistant Isolates of Acinetobacter baumannï Isolated from Urinary Tract Infections in Diyala City
}

\author{
Izdehar M. Jasim \\ Department of Biology, College of Science, University of Diyala, Baqubah, Iraq
}

\begin{abstract}
The detection of $\beta$-lactam-resistant $A$. cinetobacter baumannii in Diyala city and their resistance genetic mechanisms was undertaken. We studied the extended-spectrum beta-lactamase genes, particularly the PER-1 gene, among $\beta$-lactam-resistant $A$. baumannii isolates from patients from patients suffering from urinary tract infections in teaching Baquba hospital and Al-Betul hospital in Diyala city. From April to October (2015), 250 urine samples were collected. And the results of bacterial culture on media of MacConkey agar, blood agar and biochemical tests and confirm the diagnosis using Api $20 \mathrm{E}$ system and Vitek 2 compact system showed that 8 isolates are belonging to bacteria of Acinetobacte baumannii. Fresh subculture samples were tested for antimicrobial susceptibility against (11) antibiotics using disk diffusion method, the results revealed that all isolates were variable resistance to ampicillin, aztreonam, ceftazidium, cefotaxime, cefepime, ciprofloxacin, piperacillin, meropenem, nalidixic acid and amikacin $(62.5,50,75,75,50,50,75,37.5,50$ and $25 \%)$, respectively. Whearase all isolates were sensitive to antibiotic imipenem. Total genomic DNA was extracted from each isolate and further used for Polymerase Chain Reaction (PCR). The results of detection of $\beta$-lactam resistance gene by molcular technique PCR revealed that of these 8 isolates, 6 were found to harboring PER-1 gene.
\end{abstract}

Key words: Acinetobacter baumannii, multidrug resistant, PER-1 gene, antibiotics, technique, culture

\section{INTRODUCTION}

Acinetobacter baumannii has appeared as an main nosocomial pathogen. This organism has become endemic in some of hospital outbreaks have been described from various environmental areas. The role of the environmental corruption in the transmission of nosocomial infections in general and in $A$. baumannii infections in real is fine documented (McConnell et al., 2013; Sengstock et al., 2010). A. baumannii is Gram negative bacterium and is a typically short, almost round, rod-shaped (coccobacillus). It can be an opportunistic pathogen in peoples with compromised immune systems (Antunes et al., 2014). A. baumannii is the best described among the genus Acinetobacter and most often associated with human diseases and fatalities and viewed as an opportunistic pathogen, mostly targets liable hosts where it causes ventilator-associated pneumonia, Urinary Tract Infections (UTIs), skin, soft tissue and wound infections, secondary meningitis and bacteremia (Perez et al., 2007; Safari et al., 2013). In the 1970s, A. baumannii was liable to most antimicrobial causes. It has now become a major cause of nosocomial infection global because of its incredible ability to acquire resistance determinants to various kinds of antimicrobial means (Dijkshoorn et al., 2007). A major increase in the number and severity of cases of $A$. baumannii infections from hospital outbreaks as well as sporadic community-associated and wound-associated cases has been observed and consequently. In latest $A$. baumannii has developed as a major cause of nosocomial infections linked with significant morbidity and mortality, particularly in severely compromised individuals, ICU patients and military personnel suffering from traumatic injury and in a prevalence study of infections in ICUs showed among 75 countries of the five continents. A. baumannii was found to be the fifth most common pathogen (Vincent et al., 2009). Additionally and because the progress resistance to most antimicrobial agents, many $A$. baumannii infections can be quite severe with mortality rates ranging from 26-68\%. Moreover, Multi-Drug Resistant (MDR) strains have recently emerged globally, accentuating the need for new therapeutic attitudes for the treatment of Acinetobacter infections (McConnell et al., 2013). Gram-negative bacteria have developed increasingly resistant to antimicrobial means. They have developed several mechanisms by which they can withstand to antimicrobials, these mechanisms include the production of Extended Spectrum $\beta$-Lactamases (ESBLs) and carbapenemases (El Salabi et al., 2013).

Penicillins and cephalosporins efficiently hydrolyzes via. PER-1 $\beta$-lactamase and is susceptible to clavulanic acid inhibition. PER-1 was first noticed in $P$. aeruginosa 
isolate from a Turkish patient in France (Nordmann et al., 1993; Neuhauser et al., 2003) and later in S. enterica serovar Typhimurium and Acinetobacter isolates as well (Vahaboglu et al., 1997). In Turkey, as many as $46 \%$ of nosocomial isolates of Acinetobacter spp. and $11 \%$ of $P$. aeruginosa were establish to produce PER-1 (Vahaboglu et al., 2001). PER-2 which dividends $86 \%$ homology to PER-1 has been noticed in S. entericaserovar Typhimurium, E.coli, K. pneumoniae, $P$. mirabilis and $V$. cholera $\mathrm{O} 1 \mathrm{El}$ Tor. PER-2 has only been establish in South America, thus far (Bauernfeind et al., 1996).

While PER-1-producing organisms have been predominantly found in Turkey, a $P$. aeruginosa outbreak in Italy occurred with no apparent associates with Turkey (https://aac.asm.org/content/40/3/616.short 2001). Also PER-1 gene has also been found in P. mirabilis and Alcaligenes faecalis in Italy (Pereira et al., 2000). $P$. aeruginosa isolates producing PER-1 have been identified in France, Italy and Belgium (De Champs et al., 2002). Moreover, a high incidence of PER-1 in Acinetobacter spp. from Korea has been distinguished (Kwon et al., 2002). Also, the prevalence and genetic variety of extended-spectrum $\beta$-lactamase genes and their resistance genetic mechanisms, particularly the PER-1 gene, among carbapenem-resistant $A$. baumannii strains from patients at a tertiary care hospital in Riyadh, Saudi Arabia (Aly et al., 2016).

\section{MATERIALS AND METHODS}

Bacterial samples: A total El Salabi et al. (2013) A. baumannii isolates were isolated from UTIs by culturing urine sample on MacConkey agar, after $24 \mathrm{~h}$ of incubation at $37^{\circ} \mathrm{C}$, the suspicious colonies which were non lactose fermentative and were pale on MacConkey agar, traditional biochemical tests were used for final identification of bacterial isolates and the confirmed identifications to species level were also carried out by using Api 20 E system (Enterobacteriacea identification system, Biomrerieux, France) and by Vitek 2 system (Biomerieux, France)(MacFaddin, 2000).

The susceptibility of $A$. baumannii isolates against Ampicillin $(30 \mu \mathrm{g})$, aztreonam $(30 \mu \mathrm{g})$, ceftazidium $(10 \mu \mathrm{g})$, cefotaxime $(5 \mu \mathrm{g})$, cefepime $(30 \mu \mathrm{g})$, ciprofloxacin $(5 \mu \mathrm{g})$, piperacillin $(30 \mu \mathrm{g})$, meropenem $(10 \mu \mathrm{g})$, nalidixic acid (30 $\mu \mathrm{g})$ and amikacin $(30 \mu \mathrm{g})$ were tested using the standard disk diffusion method on Mueller Hinton (MH) agar plates and using the breakpoints defined by Clinical and Laboratory Standards Institute (2012) (CLSI., 2012).

Detection of $\boldsymbol{\beta}$-lactam resistance PER-1 gene: Total DNA was extracted from all 8 isolates by using DNA extraction kit (Bioneer, Korea) and $3 \mu \mathrm{L}$ of the isolated DNA was subjected to PCR with specific primers, PCR amplification of the PER-1 gene was with primers PER-1(foreword) 5-ATGAATGTCATTATAAAAGC-3 andPER-1 (reverse), 5-AATTTGGGCTTAGGGCAGAA-3, yielding a926-bp product. The quantity and quality of the extracted DNA were evaluated using a Nano Drop ND-1000 spectrophotometer (Thermo Fisher Scientific, Waltham, MA, USA). The DNA concentration and the ratio of the optical density at $260 / 280 \mathrm{~nm}$ to evaluate the purity of the DNA samples were calculated simultaneously. Conventional PCR reactions with genomic DNA were achieved in a $25 \mu \mathrm{L}$ mixture according to the manufacturer's protocol for the Maxima SYBR Green/ GoTaqPCR Master Mix (Thermo Fisher Scientific).

PCR conditions were $10 \mathrm{~min}$ at $94 \mathrm{C}$, followed by 35 cycles of $1 \mathrm{~min}$ at $94^{\circ} \mathrm{C}, 1 \mathrm{~min}$ at $55^{\circ} \mathrm{C}$ and $1 \mathrm{~min}$ at $72^{\circ} \mathrm{C}$, followed by a final extension for $10 \mathrm{~min}$ at $72^{\circ} \mathrm{C}$. Agarose gel electrophoresis was carried out in conventional Tris-Borate-EDTA (TBE) buffer with agarose $1 \% \mathrm{~W} / \mathrm{V}$ for PER-1-PCR product,. A 100 bp DNA ladder mix (Promega/USA) was used to provide ë molecular size markers. The gels were stained with ethidium bromide and observed under UV transillumination.

\section{RESULTS AND DISCUSSION}

Disk diffusion test was employed to determine antibiotic susceptibility of $A$. baumannii isolates on MHA following the Clinical and Laboratory Standards Institute (CLSI., 2012) guideline. The results revealed that all isolates were variable resistance to ampicillin, aztreonam, ceftazidium, cefotaxime, cefepime, ciprofloxacin, piperacillin, meropenem, nalidixic acid and amikacin $(62.5$, $50,75,75,50,50,75,37.5,50$ and $25 \%$ ), respectively. Whearase all isolates were sensitive to antibiotic imipenem. Traditionally, infections were treated with imipenem or meropenem but a steady rise in carbapenem-resistant $A$. baumannii has been noted (Su et al., 2012). Prevention methods in hospitals focus on increased hand-washing and more diligent sterilization actions (Anonymous, 2013). The current study with agreement with previous Iraqi studies (Al-Muhanna, 2006) make study about Acinetobacter spp. And find that all isolates were sensitive to imipenem (Al-Muhanna, 2006) and Al-Ajeeli also find all $A$. boumannii isolates were sensitive to imipenem (Al-Ajeeli, 2014). Whearse study by Al-Bajlani revealed that $50 \%$ of $A$. boumannii isolates were resist to meropenem and imipenem (Al-Bajlany, 2015).

The development of resistance to the carbapenem group belong to produce carbapenemases, also Siroy et al. (2005) observed that the lost of $29 \mathrm{kDa}$ protein in the outer membrane of bacteria that defined as Car $O$ which have relation with the resistance to meropenem and 


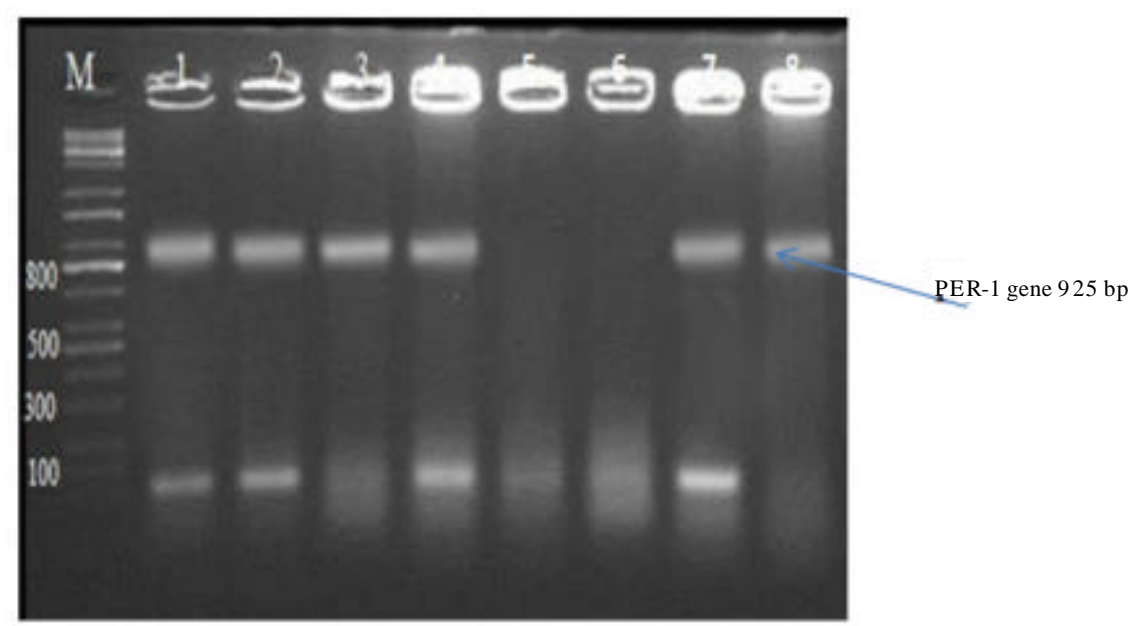

Fig. 1: Agarose gel electrophoresis of PCR amplification products of, blaPER-1 gene (1.5\% agarose, TBE buffer $(1 \mathrm{X}), 7 \mathrm{~V} / \mathrm{cm}, 90 \mathrm{~min})$. M: The DNA molecular wight marker (100 bp ladder). All lanes positive amplification of 925 bp for blaPER-1 gene except lane 5 and 6 are negative

imipenem (Siroy et al., 2005). Also, the current study detect that $6(75 \%)$ isolates of $A$. boumannii were found to be resist to ceftazidium, cefotaxime and piperacillin and that in agreement with studies by Al-Bajlany (2015) and Al-Masoudi et al. (2015).

The PCR amplification results of nine isolates of $A$. baumannii revealed that the extended-spectrum $\beta$-lactamase blaPER-1 gene was present in six isolates. PCR amplified blaPER-1 gene showed a molecular weight of 925 bp, Fig. 1 the study by Kim et al. (2008) exposed that 42 Multidrug-Resistant (MDR) A. baumannii isolates were attained throughout outbreaks in a Korean hospital. The co-carriage of blaOXA-23, blaOXA-51, blaPER-1 and armA was observed in 23 isolates (Kim et al., 2008; Sacha et al., 2012) detected that PCR technique did not approve the presence of the blaPER-1 gene in any of the $A$. baumannii strains observed in their hospital. A. baumannii strains reveal significant resistance to many groups of antibiotics (Sacha et al., 2012). The current study in agreement with study by Aly et al. (2016) genotyping results of PER-1-like genes showed that 384/503 (76.3\%) were positive among MDR Acinetobacter isolates (Aly et al., 2016). Wherase Cao et al. (2009), observed that among the 64 MDRA isolates 39.1\% (Al-Bajlany, 2015) with blaPER-1 gene.

\section{CONCLUSION}

The prevalence of multidrug-resistant $A$. baumannii occurred at 2 hospitals with the prevalence of the PER-1 resistance gene among them in percentage $(75 \%)$.

\section{REFERENCES}

AL-Masoudi, K.K., J.M. AL-Saffar and N.J. Kendla, 2015. Molecular characteristics of multidrug resistant Acinetobacter baumannii Isolated from Baghdad Hospitals. Iraqi J. Sci., 56: 1394-1399.

Al-Ajeeli, S.S.M., 2014. Molculardetection of genomic islands responsible for Antimicrobial resistance in Acinetobacter baumannii Strain A92. Ph.D Thesis, University of Baghdad, Baghdad, Iraq.

Al-Bajlany, S.M.M., 2015. Detection of extended spectrum beta-lactam resistance genes in Acinetobacter baumannii isolated from clinical sources. MSc Thesis, University of Diayla, Baqubah, Iraq.

Al-Muhanna, A.S.J., 2006. A molcular and genetic study of virulence factors of Acinetobacter isolated from different infections. Ph.D Thesis, Al-Mustansiriya University, Baghdad, Iraq.

Aly, M.M., N.A. Alsoud, M.S. Elrobh, S.M. Al Johani and H.H. Balkhy, 2016. High prevalence of the PER-1 gene among carbapenem-resistant Acinetobacter baumannii in Riyadh, Saudi Arabia. Eur. J. Clin. Microbiol. Infect. Dis., 35: 1759-1766.

Anonymous, 2013. Acinetobacter in healthcare settings. Centers for Disease Control and Prevention, USA.

Antunes, L.C.S., P. Visca and K.J. Towner, 2014. Acinetobacter baumannit: Evolution of a global pathogen. Pathogens Dis., 71: 292-301. 
Bauernfeind, A., I. Stemplinger, R. Jungwirth, P. Mangold and S. Amann et al., 1996. Characterization of beta-lactamase gene blaPER-2 which encodes an extended-spectrum class a beta-lactamase. Antimicrob. Agents Chemother., 40: 616-620.

CLSI., 2012. Methods for dilution antimicrobial susceptibility tests for bacteria that grow aerobically. Approved Standard M7-A9, Clinical and Laboratory Standards Institute, Wayne, PA., USA.

Cao, J.R., X. Wei, Z.Q. Yan, D.X. Shen and Y.P. Luo, 2009. Study on the molecular characteristics of multidrug-resistant Acinetobacter baumannii. Zhonghua Liuxingbingxue Zazhi, 30: 832-835.

De Champs, C., L. Poirel, R. Bonnet, D. Sirot and C. Chanal et al., 2002. Prospective survey of B-lactamases produced by ceftazidime-resistant Pseudomonas aeruginosa isolated in a French hospital in 2000. Antimicrob. Agents Chemother., 46: 3031-3034.

Dijkshoorn, L., A. Nemec and H. Seifert, 2007. An increasing threat in hospitals: Multidrug-resistant Acinetobacter baumannii. Nat. Rev. Microbiol., 5: 939-951.

El Salabi, A., T.R. Walsh and C. Chouchani, 2013. Extended spectrum ß-lactamases, carbapenemases and mobile genetic elements responsible for antibiotics resistance in Gram-negative bacteria. Crit. Rev. Microbial., 39: 113-122.

Kim, J.W., S.T. Heo, J.S. Jin, C.H. Choi and Y.C. Lee et al., 2008. Characterization of Acinetobacter baumannii carrying blaOXA.23blaPER-1 and armA in a Korean Hospital. Clin. Microbial. Infect., 14: 716-718.

Kwon, N.Y., J.D. Kim and H.J. Pai, 2002. The resistance mechanisms of b-lactam Antimicrobials in clinical isolates of Acinetobacter baumannii. Korean J. Internal Med., 17: 94-99.

MacFaddin, J.F., 2000. Biochemical Tests for Identification of Medical Bacteria. 3rd Edn., Lippincott Williams \& Wilkins, London, UK., Pages: 912.

McConnell, M.J., L. Actis and J. Pachon, 2013. Acinetobacter baumannit: human infections, factors contributing to pathogenesis and animal models. FEMS Microbiol. Rev., 37: 130-155.

Neuhauser, M.M., R.A. Weinstein, R. Rydman, L.H. Danziger and G. Karam et al., 2003. Antibiotic resistance among Gram-negative bacilli in US intensive care units: Implications for fluoroquinolone use. Jama, 289: 885-888.
Nordmann, P., S. Mariotte, T. Naas, R. Labia and M.H. Nicolas, 1993. Biochemical properties of a carbapenem hydrolyzing $\beta$-lactamase from Enterobacter cloacae and cloning of the gene into Escherichia coli. Antimicrob. Agents Chemother., 37: 939-946.

Pereira, M., M. Perilli, E. Mantengoli, F. Luzzaro and A. Toniolo et al., 2000. PER-1 extended-spectrum B-lactamase production in an Alcaligenes faecalis clinical isolate resistant to expanded-spectrum Cephalosporins and monobactams from a hospital in Northern Italy. Microb. Drug Resist., 6: 85-90.

Perez, F., A.M. Hujer, K.M. Hujer, B.K. Decker, P.N. Rather and R.A. Bonomo, 2007. Global challenge of multidrug-resistant Acinetobacter baumannii. Antimicrob. Agents Chemother., 51: 3471-3484.

Sacha, P., P. Wieczorek, D. Ojdana, S. Czaban and W Klosowska et al., 2012. Susceptibility, phenotypes of resistance and extended-spectrum ß-lactamases in Acinetobacter baumannii strains. Folia Histochemica Cytobiologica, 50: 46-51.

Safari, M., M. Saidijam, A. Bahador, R. Jafari and M.Y. Alikhani, 2013. High prevalence of multidrug resistance and Metallo-beta-Lactamase (MßL) producing Acinetobacter baumannii isolated from patients in ICU wards, Hamadan, Iran. J. Res. Health Sci., 13: 162-165.

Sengstock, D.M., R. Thyagarajan, J. Apalara, A. Mira and T. Chopra et al., 2010. Multidrug-resistant Acinetobacter baumannii: An emerging pathogen among older adults in community hospitals and nursing homes. Clin. Infect. Dis., 50: 1611-1616.

Siroy, A., V. Molle, C. Lemaitre-Guillier, D. Vallenet and M. Pestel-Caron et al., 2005. Channel formation by $\mathrm{CarO}$, the carbapenem resistance-associated outer membrane protein of Acinetobacter baumannii. Antimicrob. Agents Chemother., 49: 4876-4883.

Su, C.H., J.T. Wang, C.A. Hsiung, L.J. Chien and C.L. Chi et al., 2012. Increase of carbapenem-resistant Acinetobacter baumannii infection in acute care hospitals in Taiwan: Association with hospital antimicrobial usage. PloS One, 7: 1-6.

Vahaboglu, H., F. Coskunkan, O. Tansel, R. Ozturk and N. Sahin et al., 2001. Clinical importance of extended-spectrum B-lactamase (PER-1-type)producing Acinetobacter spp. and Pseudomonas aeruginosa strains. J. Med. Microbial., 50: 642-645.

Vahaboglu, H., R. Ozturk, G. Aygun, F. Coskunkan and A. Yaman et al., 1997. Widespread detection of PER-1-type extended-spectrum beta-lactamases among nosocomial acinetobacter and Pseudomonas aeruginosa isolates in Turkey: A nationwide multicenter study. Antimicrobiol. Agents Chemother., 41: 2265-2269. 
Vincent, J.L., J. Rello, J. Marshall, E. Silva and A. Anzueto et al., 2009. International study of the prevalence and outcomes of infection in intensive care units. J. Am. Med. Assoc., 302: 2323-2329. https: //aac.asm.org/content/40/3/616.short2001. Dynamics of a nosocomial outbreak of multidrug-resistant Pseudomonas aeruginosa producing the PER-1 extended-spectrum B-lactamase. J. Clin. Microbial., 39: $1865-1870$. 